\title{
On the Chebyshev polynomials and some of their new identities
}

Di Han ${ }^{1}$ and Xingxing $L V^{1 *}$ CD

"Correspondence:

Ivxingxing@stumail.nwu.edu.cn

${ }^{1}$ School of Mathematics, Northwest

University, Xi'an, P.R. China

\section{Springer}

\begin{abstract}
The main purpose of this paper is, using the elementary methods and properties of the power series, to study the computational problem of the convolution sums of Chebyshev polynomials and Fibonacci polynomials and to give some new and interesting identities for them.
\end{abstract}

MSC: 11B39; $11 \mathrm{~B} 83$

Keywords: Chebyshev polynomials; Fibonacci polynomials; Lucas polynomials; Power series; Convolution sums; New identity

\section{Introduction}

For any integer $n \geq 0$, the famous Chebyshev polynomial of the first kind $\left\{T_{n}(x)\right\}$ is defined as follows:

$$
T_{n+2}(x)=2 x T_{n+1}(x)-T_{n}(x) \quad \text { for all integers } n \geq 0 \text {, with } T_{0}(x)=1, T_{1}(x)=x \text {. }
$$

Let $\alpha=x+\sqrt{x^{2}-1}$ and $\beta=x-\sqrt{x^{2}-1}$ be two characteristic roots of the equation $\lambda^{2}-$ $2 x \lambda+1=0$, then we have (see [1] and [2])

$$
T_{n}(x)=\frac{1}{2}\left(\alpha^{n}+\beta^{n}\right), \quad U_{n}(x)=\frac{\alpha^{n+1}-\beta^{n+1}}{\delta-\beta}, \quad n=0,1,2, \ldots,
$$

where $\left\{U_{n}(x)\right\}$ is Chebyshev polynomial of the second kind with $U_{0}(x)=1$ and $U_{1}(x)=2 x$. The generating functions of $T_{n}(x)$ and $U_{n}(x)$ are

$$
\frac{1-x t}{1-2 x t+t^{2}}=\sum_{n=0}^{\infty} T_{n}(x) \cdot t^{n} \quad \text { and } \quad \frac{1}{1-2 x t+t^{2}}=\sum_{n=0}^{\infty} U_{n}(x) \cdot t^{n}
$$

For convenience, we also extend the recursive properties of $T_{n}(x)$ and $U_{n}(x)$ in (1) to all negative integers.

We all know that the polynomials $T_{n}(x)$ and $U_{n}(x)$ play important roles in the study of orthogonality of functions and approximation theory, so many scholars have studied their properties and obtained a series of valuable research results. In particular, in the references

c) The Author(s) 2020. This article is licensed under a Creative Commons Attribution 4.0 International License, which permits use, sharing, adaptation, distribution and reproduction in any medium or format, as long as you give appropriate credit to the original author(s) and the source, provide a link to the Creative Commons licence, and indicate if changes were made. The images or other third party material in this article are included in the article's Creative Commons licence, unless indicated otherwise in a credit line to the material. If material is not included in the article's Creative Commons licence and your intended use is not permitted by statutory regulation or exceeds the permitted use, you will need to obtain permission directly from the copyright holder. To view a copy of this licence, visit http://creativecommons.org/licenses/by/4.0/. 
we have seen that Kim and his team have done a lot of important research work (see [3$11]$ ), and Cesarano (see [12-14]) has also made a lot of contributions. Some other papers related to these polynomials and sequences can be found in references [2, 15-29]. For example, Zhang Wenpeng [17] studied the calculating problem of the convolution sums of $T_{n}(x)$ and proved the following:

$$
\begin{gathered}
\sum_{a_{1}+a_{2}+\cdots+a_{k+1}=n+k+1} T_{a_{1}}(x) \cdot T_{a_{2}}(x) \cdot T_{a_{3}}(x) \cdots T_{a_{k+1}}(x) \\
=\frac{1}{2^{k} \cdot k !} \sum_{h=0}^{k+1}(-x)^{h}\left(\begin{array}{c}
k+1 \\
h
\end{array}\right) U_{n+2 k+1-h}^{(k)}(x),
\end{gathered}
$$

where $\sum_{a_{1}+a_{2}+\cdots+a_{k+1}=n}$ denotes that the summation is taken over all $(k+1)$-dimension nonnegative integer coordinates $\left(a_{1}, a_{2}, \ldots, a_{k+1}\right)$ such that $a_{1}+a_{2}+\cdots+a_{k}+a_{k+1}=n$, $U_{n}^{(k)}(x)$ denotes the $k$ th derivative of $U_{n}(x)$ with respect to $x$.

Zhang Yixue and Chen Zhuoyu [18] proved the following result:

$$
\begin{aligned}
& \quad \sum_{a_{1}+a_{2}+\cdots+a_{h+1}=n} U_{a_{1}}(x) U_{a_{2}}(x) \cdots U_{a_{h+1}}(x) \\
& =\frac{1}{2^{h} \cdot h !} \cdot \sum_{j=1}^{h} \frac{C(h, j)}{x^{2 h-j}} \sum_{i=0}^{n} \frac{(n-i+j) !}{(n-i) !} \cdot\left(\begin{array}{c}
2 h+i-j-1 \\
i
\end{array}\right) \cdot \frac{U_{n-i+j}(x)}{x^{i}},
\end{aligned}
$$

where $C(h, i)$ is a second-order nonlinear recurrence sequence defined by $C(h, 0)=0$, $C(h, h)=1, C(h+1,1)=1 \cdot 3 \cdot 5 \cdots(2 h-1)=(2 h-1) ! !$, and $C(h+1, i+1)=(2 h-1-$ i) $\cdot C(h, i+1)+C(h, i)$ for all $1 \leq i \leq h-1$.

Obviously the results in $[17,18]$, and $[21]$ do not look very concise and clear. It is even harder to calculate their exact values. Inspired by these papers, we also became interested in such problems and used different methods to come up with simpler, more beautiful identities. That is, we use the elementary methods and the properties of the power series to prove the following conclusions:

Theorem 1 For any integers $k \geq 2$ and $n \geq 0$, we have the identity

$$
\begin{aligned}
& \quad \sum_{a_{1}+a_{2}+\cdots+a_{k}=n} T_{a_{1}}(x) \cdot T_{a_{2}}(x) \cdot T_{a_{3}}(x) \cdots T_{a_{k}}(x) \\
& =\frac{1}{2^{k-1}}\left(\begin{array}{c}
n+k-1 \\
k-1
\end{array}\right) T_{n}(x) \\
& \quad+\frac{1}{2^{k}} \sum_{i=0}^{n}\left(\sum_{h=1}^{k-1}\left(\begin{array}{l}
k \\
h
\end{array}\right)\left(\begin{array}{c}
i+h-1 \\
h-1
\end{array}\right)\left(\begin{array}{c}
n-i+k-h-1 \\
k-h-1
\end{array}\right)\right) \cdot T_{n-2 i}(x) .
\end{aligned}
$$

Theorem 2 For any integer $k \geq 2$ and integer $n \geq 0$, we have

$$
\begin{array}{r}
\sum_{a_{1}+a_{2}+\cdots+a_{k}=n} U_{a_{1}}(x) \cdot U_{a_{2}}(x) \cdot U_{a_{3}}(x) \cdots U_{a_{k}}(x) \\
=\sum_{i=0}^{n}\left(\begin{array}{c}
i+k-1 \\
k-1
\end{array}\right)\left(\begin{array}{c}
n-i+k-1 \\
k-1
\end{array}\right) \cdot T_{n-2 i}(x) .
\end{array}
$$


For Lucas polynomials $L_{n+2}(x)=x L_{n+1}(x)+L_{n}(x)$ with $L_{0}(x)=2, L_{1}(x)=x$ and Fibonacci polynomials $F_{n+2}=x F_{n+1}+F_{n}(x)$ with $F_{0}(x)=0$ and $F_{1}(x)=1$, we can also deduce the following corresponding results:

Theorem 3 For any positive integer $k \geq 2$ and integer $n \geq 0$, we have

$$
\begin{aligned}
& \sum_{a_{1}+a_{2}+\cdots+a_{k}=n} L_{a_{1}}(x) \cdot L_{a_{2}}(x) \cdots L_{a_{k}}(x) \\
& =\left(\begin{array}{c}
n+k-1 \\
k-1
\end{array}\right) \cdot L_{n}(x) \\
& \quad+\frac{1}{2} \sum_{i=0}^{n}(-1)^{i} \cdot\left(\sum_{h=1}^{k-1}\left(\begin{array}{l}
k \\
h
\end{array}\right)\left(\begin{array}{c}
i+h-1 \\
h-1
\end{array}\right)\left(\begin{array}{c}
n-i+k-h-1 \\
k-h-1
\end{array}\right)\right) L_{n-2 i}(x) .
\end{aligned}
$$

Theorem 4 For any positive integer $k \geq 2$ and integer $n \geq 0$, we have

$$
\begin{aligned}
& \sum_{a_{1}+a_{2}+\cdots+a_{k}=n} F_{a_{1}}(x) \cdot F_{a_{2}}(x) \cdot F_{a_{3}}(x) \cdots F_{a_{k}}(x) \\
& =\sum_{i=0}^{n}(-1)^{i}\left(\begin{array}{c}
i+k-1 \\
k-1
\end{array}\right)\left(\begin{array}{c}
n-i+k-1 \\
k-1
\end{array}\right) L_{n-2 i}(x) .
\end{aligned}
$$

Note the identity $L_{n}(x)=F_{n+1}(x)+F_{n-1}(x)$, it is clear that our Theorem 4 is much simpler than the corresponding identity in Ma Yuankui and Zhang Wenpeng [20]. Taking $k=3,4$ or $k=5$ with $x=1$, from Theorems 2 and 4 we can deduce the following four corollaries:

Corollary 1 For any integer $n \geq 0$, we have the identity

$$
\begin{aligned}
& \sum_{a+b+c+d=n} U_{a}(x) \cdot U_{b}(x) \cdot U_{c}(x) \cdot U_{d}(x) \\
& =\frac{1}{36} \sum_{i=0}^{n}(i+1)(i+2)(i+3)(n-i+1)(n-i+2)(n-i+3) \cdot T_{n-2 i}(x)
\end{aligned}
$$

Corollary 2 For any integer $n \geq 0$, we have the identity

$$
\begin{aligned}
& \sum_{a+b+c=n} F_{a}(x) \cdot F_{b}(x) \cdot F_{c}(x) \\
& \quad=\frac{1}{4} \sum_{i=0}^{n}(-1)^{i}(i+1)(i+2)(n-i+1)(n-i+2) \cdot L_{n-2 i}(x) .
\end{aligned}
$$

Corollary 3 For any integer $n \geq 0$, we have the identity

$$
\begin{aligned}
& \sum_{a+b+c+d=n} F_{a}(x) \cdot F_{b}(x) \cdot F_{c}(x) \cdot F_{d}(x) \\
& =\frac{1}{36} \sum_{i=0}^{n}(-1)^{i}(i+1)(i+2)(i+3)(n-i+1)(n-i+2)(n-i+3) L_{n-2 i}(x) .
\end{aligned}
$$


Corollary 4 For any integer $n \geq 0$, we have the identity

$$
\sum_{a+b+c+d+e=n} F_{a+1} \cdot F_{b+1} \cdot F_{c+1} \cdot F_{d+1} \cdot F_{e+1}=\sum_{i=0}^{n}(-1)^{i}\left(\begin{array}{c}
i+4 \\
4
\end{array}\right)\left(\begin{array}{c}
n-i+4 \\
4
\end{array}\right) L_{n-2 i} .
$$

For any nonnegative integers $m$ and $n$, note that $T_{m}\left(T_{n}(x)\right)=T_{m n}(x)$. From Theorem 1 we can also deduce the following:

Corollary 5 For any integers $k \geq 2$ and $m \geq 0$, we have the identity

$$
\begin{aligned}
& \sum_{a_{1}+a_{2}+\cdots+a_{k}=n} T_{m a_{1}}(x) \cdot T_{m a_{2}}(x) \cdot T_{m a_{3}}(x) \cdots T_{m a_{k}}(x) \\
& =\frac{1}{2^{k-1}}\left(\begin{array}{c}
n+k-1 \\
k-1
\end{array}\right) T_{m n}(x) \\
& \quad+\frac{1}{2^{k}} \sum_{i=0}^{n}\left(\sum_{h=1}^{k-1}\left(\begin{array}{l}
k \\
h
\end{array}\right)\left(\begin{array}{c}
i+h-1 \\
h-1
\end{array}\right)\left(\begin{array}{c}
n-i+k-h-1 \\
k-h-1
\end{array}\right)\right) \cdot T_{m(n-2 i)}(x) .
\end{aligned}
$$

Some notes: It is worth noting that Theorem 1 has been obtained by different methods in equation (29) of [9], but the expression is different from our result. In fact equation (29) in [9] involved the Gauss hypergeometric function, so it looks a little bit more complicated, and our Theorem 1 is simple and straightforward. Theorem 2 has been obtained by different methods in (1.30) of [3].

\section{Proofs of the theorems}

Now we prove our main results directly. First, we will prove Theorem 1. From the generating function (2) of $T_{n}(x)$, we have

$$
\frac{1}{2}\left[\frac{1}{1-\alpha t}+\frac{1}{1-\beta t}\right]=\frac{1-x t}{1-2 x t+t^{2}}=\sum_{n=0}^{\infty} T_{n}(x) \cdot t^{n} .
$$

So, for any positive integer $k \geq 2$, from (3) and the properties of the power series, we have the identity

$$
\frac{1}{2^{k}}\left[\frac{1}{1-\alpha t}+\frac{1}{1-\beta t}\right]^{k}=\sum_{n=0}^{\infty}\left(\sum_{a_{1}+a_{2}+\cdots+a_{k}=n} T_{a_{1}}(x) \cdot T_{a_{2}}(x) \cdots T_{a_{k}}(x)\right) \cdot t^{n} .
$$

On the other hand, note the power series

$$
\frac{1}{(1-x)^{k}}=\sum_{n=0}^{\infty}\left(\begin{array}{c}
n+k-1 \\
k-1
\end{array}\right) \cdot x^{n}, \quad|x|<1
$$

For any positive integers $r$ and $h$, we have

$$
\begin{aligned}
& \frac{1}{(1-\alpha t)^{r}(1-\beta t)^{h}} \\
& =\left(\sum_{n=0}^{\infty}\left(\begin{array}{c}
n+r-1 \\
r-1
\end{array}\right) \alpha^{n} t^{n}\right)\left(\sum_{n=0}^{\infty}\left(\begin{array}{c}
n+h-1 \\
h-1
\end{array}\right) \beta^{n} t^{n}\right)
\end{aligned}
$$




$$
\begin{aligned}
& =\sum_{n=0}^{\infty}\left(\sum_{i=0}^{n}\left(\begin{array}{c}
i+r-1 \\
r-1
\end{array}\right)\left(\begin{array}{c}
n-i+h-1 \\
h-1
\end{array}\right) \cdot \alpha^{i} \cdot \beta^{n-i}\right) \cdot t^{n} \\
& =\sum_{n=0}^{\infty}\left(\sum_{i=0}^{n}\left(\begin{array}{c}
i+r-1 \\
r-1
\end{array}\right)\left(\begin{array}{c}
n-i+h-1 \\
h-1
\end{array}\right) \cdot \beta^{n-2 i}\right) \cdot t^{n}
\end{aligned}
$$

where $\alpha \cdot \beta=1$.

From (6) and the definition of $T_{n}(x)$, we have

$$
\begin{aligned}
& \frac{1}{2}\left[\frac{1}{(1-\alpha t)^{r}(1-\beta t)^{h}}+\frac{1}{(1-\alpha t)^{h}(1-\beta t)^{r}}\right] \\
& \quad=\sum_{n=0}^{\infty}\left(\sum_{i=0}^{n}\left(\begin{array}{c}
i+r-1 \\
r-1
\end{array}\right)\left(\begin{array}{c}
n-i+h-1 \\
h-1
\end{array}\right) \cdot \frac{1}{2}\left(\alpha^{n-2 i}+\beta^{n-2 i}\right)\right) \cdot t^{n} \\
& \quad=\sum_{n=0}^{\infty}\left(\sum_{i=0}^{n}\left(\begin{array}{c}
i+r-1 \\
r-1
\end{array}\right)\left(\begin{array}{c}
n-i+h-1 \\
h-1
\end{array}\right) \cdot T_{n-2 i}(x)\right) \cdot t^{n} .
\end{aligned}
$$

From (5), (7), the definition and properties of the binomial, we have

$$
\begin{aligned}
&\left(\frac{1}{1-\alpha t}+\frac{1}{1-\beta t}\right)^{k} \\
&=\sum_{h=0}^{k}\left(\begin{array}{l}
k \\
h
\end{array}\right) \frac{1}{(1-\alpha t)^{h}} \cdot \frac{1}{(1-\beta t)^{k-h}} \\
&=\frac{1}{(1-\alpha t)^{k}}+\frac{1}{(1-\beta t)^{k}} \\
&+\frac{1}{2} \sum_{h=1}^{k-1}\left(\frac{\left(\begin{array}{l}
k \\
h
\end{array}\right)}{(1-\alpha t)^{h}(1-\beta t)^{k-h}}+\frac{\left(\begin{array}{c}
k \\
h
\end{array}\right)}{(1-\alpha t)^{k-h}(1-\beta t)^{h}}\right) \\
&=\sum_{h=1}^{k-1}\left(\begin{array}{l}
k \\
h
\end{array}\right) \sum_{n=0}^{\infty}\left(\begin{array}{l}
n \\
i=0
\end{array}\left(\begin{array}{c}
i+h-1 \\
h-1
\end{array}\right)\left(\begin{array}{c}
n-i+k-h-1 \\
k-h-1
\end{array}\right) \cdot T_{n-2 i}(x)\right) \cdot t^{n} \\
&+2 \sum_{n=0}^{\infty}\left(\begin{array}{c}
n+k-1 \\
k-1
\end{array}\right) \cdot T_{n}(x) \cdot t^{n} \\
&= \sum_{n=0}^{\infty}\left(\begin{array}{l}
k-1 \\
h=1
\end{array}\left(\begin{array}{l}
k \\
h
\end{array}\right)\left(\sum_{i=0}^{n}\left(\begin{array}{c}
i+h-1 \\
h-1
\end{array}\right)\left(\begin{array}{c}
n-i+k-h-1 \\
k-h-1
\end{array}\right) \cdot T_{n-2 i}(x)\right)\right) \cdot t^{n} \\
&+2 \sum_{n=0}^{\infty}\left(\begin{array}{c}
n+k-1 \\
k-1
\end{array}\right) \cdot T_{n}(x) \cdot t^{n} .
\end{aligned}
$$

Combining (4) and (8) and then comparing the coefficients of the power series, we have the identity

$$
\begin{aligned}
& \sum_{a_{1}+a_{2}+a_{3}+\cdots+a_{k}=n} T_{a_{1}}(x) \cdot T_{a_{2}}(x) \cdot T_{a_{3}}(x) \cdots T_{a_{k}}(x) \\
& =\frac{1}{2^{k-1}} \cdot\left(\begin{array}{c}
n+k-1 \\
k-1
\end{array}\right) \cdot T_{n}(x)
\end{aligned}
$$




$$
+\frac{1}{2^{k}} \sum_{i=0}^{n}\left(\sum_{h=1}^{k-1}\left(\begin{array}{l}
k \\
h
\end{array}\right)\left(\begin{array}{c}
i+h-1 \\
h-1
\end{array}\right)\left(\begin{array}{c}
n-i+k-h-1 \\
k-h-1
\end{array}\right)\right) \cdot T_{n-2 i}(x) .
$$

This proves Theorem 1.

To prove Theorem 2, note that, for any positive integer $k$,

$$
\frac{1}{\left(1-2 x t+t^{2}\right)^{k}}=\sum_{n=0}^{\infty}\left(\sum_{a_{1}+a_{2}+\cdots+a_{k}=n} U_{a_{1}}(x) \cdot U_{a_{2}}(x) \cdots U_{a_{k}}(x)\right) \cdot t^{n} .
$$

From (5) and (9) we have

$$
\begin{aligned}
& \sum_{n=0}^{\infty}\left(\sum_{a_{1}+a_{2}+\cdots+a_{k}=n} U_{a_{1}}(x) \cdot U_{a_{2}}(x) \cdots U_{a_{k}}(x)\right) \cdot t^{n} \\
& =\frac{1}{(1-\alpha t)^{k} \cdot(1-\beta t)^{k}} \\
& =\left(\sum_{n=0}^{\infty}\left(\begin{array}{c}
n+k-1 \\
k-1
\end{array}\right) \cdot \alpha^{n} \cdot t^{n}\right) \cdot\left(\sum_{n=0}^{\infty}\left(\begin{array}{c}
n+k-1 \\
k-1
\end{array}\right) \cdot \beta^{n} \cdot t^{n}\right) \\
& =\sum_{n=0}^{\infty}\left(\sum_{i=0}^{n}\left(\begin{array}{c}
i+k-1 \\
k-1
\end{array}\right)\left(\begin{array}{c}
n-i+k-1 \\
k-1
\end{array}\right) \cdot \alpha^{i} \cdot \beta^{n-i}\right) \cdot t^{n} \\
& =\sum_{n=0}^{\infty}\left(\sum_{i=0}^{n}\left(\begin{array}{c}
i+k-1 \\
k-1
\end{array}\right)\left(\begin{array}{c}
n-i+k-1 \\
k-1
\end{array}\right) \cdot \beta^{n-2 i}\right) \cdot t^{n} \\
& =\frac{1}{2} \sum_{n=0}^{\infty}\left(\sum_{i=0}^{n}\left(\begin{array}{c}
i+k-1 \\
k-1
\end{array}\right)\left(\begin{array}{c}
n-i+k-1 \\
k-1
\end{array}\right) \cdot\left(\alpha^{n-2 i}+\beta^{n-2 i}\right)\right) \cdot t^{n} \\
& =\sum_{n=0}^{\infty}\left(\sum_{i=0}^{n}\left(\begin{array}{c}
i+k-1 \\
k-1
\end{array}\right)\left(\begin{array}{c}
n-i+k-1 \\
k-1
\end{array}\right) \cdot T_{n-2 i}(x)\right) \cdot t^{n} .
\end{aligned}
$$

Comparing the coefficients of $t^{n}$ in (10), we have the identity

$$
\begin{array}{r}
\sum_{a_{1}+a_{2}+\cdots+a_{k}=n} U_{a_{1}}(x) \cdot U_{a_{2}}(x) \cdot U_{a_{3}}(x) \cdots U_{a_{k}}(x) \\
=\sum_{i=0}^{n}\left(\begin{array}{c}
i+k-1 \\
k-1
\end{array}\right)\left(\begin{array}{c}
n-i+k-1 \\
k-1
\end{array}\right) \cdot T_{n-2 i}(x) .
\end{array}
$$

This proves Theorem 2 .

Let $\gamma=\frac{x+\sqrt{x^{2}+4}}{2}$ and $\delta=\frac{x-\sqrt{x^{2}+4}}{2}$, then from the definition of $L_{n}(x)$ we have $L_{n}(x)=\gamma^{n}+\delta^{n}$ and

$$
\frac{2-x t}{1-x t-t^{2}}=\frac{1}{1-\gamma t}+\frac{1}{1-\delta t}=\sum_{n=0}^{\infty} L_{n}(x) \cdot t^{n}
$$

Note that $\gamma \cdot \delta=-1$, from (5), (6), and the methods of proving Theorem 1 , we have

$$
\sum_{n=0}^{\infty}\left(\sum_{a_{1}+a_{2}+\cdots+a_{k}=n} L_{a_{1}}(x) \cdot L_{a_{2}}(x) \cdots L_{a_{k}}(x)\right) \cdot t^{n}
$$




$$
\begin{aligned}
= & \left(\frac{1}{1-\gamma t}+\frac{1}{1-\delta t}\right)^{k} \\
= & \frac{1}{(1-\gamma t)^{k}}+\frac{1}{(1-\delta t)^{k}}+\frac{1}{2} \sum_{h=1}^{k-1}\left(\frac{\left(\begin{array}{l}
k \\
h
\end{array}\right)}{(1-\gamma t)^{h}(1-\delta t)^{k-h}}+\frac{\left(\begin{array}{l}
k \\
h
\end{array}\right)}{(1-\gamma t)^{k-h}(1-\delta t)^{h}}\right) \\
= & \frac{1}{2} \sum_{n=0}^{\infty}\left(\sum_{h=1}^{k-1}\left(\begin{array}{l}
k \\
h
\end{array}\right) \sum_{i=0}^{n}\left(\begin{array}{c}
i+h-1 \\
h-1
\end{array}\right)\left(\begin{array}{c}
n-i+k-h-1 \\
k-h-1
\end{array}\right) \cdot(-1)^{i} \cdot L_{n-2 i}(x)\right) \cdot t^{n} \\
& +\sum_{n=0}^{\infty}\left(\begin{array}{c}
n+k-1 \\
k-1
\end{array}\right)\left(\gamma^{n}+\delta^{n}\right) \cdot t^{n} .
\end{aligned}
$$

Comparing the coefficients of $t^{n}$ in (12), we have

$$
\begin{aligned}
& \sum_{a_{1}+a_{2}+\cdots+a_{k}=n} L_{a_{1}}(x) \cdot L_{a_{2}}(x) \cdots L_{a_{k}}(x) \\
& =\left(\begin{array}{c}
n+k-1 \\
k-1
\end{array}\right) \cdot L_{n}(x) \\
& \quad+\frac{1}{2} \sum_{i=0}^{n}(-1)^{i} \cdot\left(\sum_{h=1}^{k-1}\left(\begin{array}{l}
k \\
h
\end{array}\right)\left(\begin{array}{c}
i+h-1 \\
h-1
\end{array}\right)\left(\begin{array}{c}
n-i+k-h-1 \\
k-h-1
\end{array}\right)\right) L_{n-2 i}(x) .
\end{aligned}
$$

This proves Theorem 3.

Similarly, from the methods of proving Theorem 2, we can also deduce

$$
\begin{aligned}
& \sum_{a_{1}+a_{2}+\cdots+a_{k}=n} F_{a_{1}}(x) \cdot F_{a_{2}}(x) \cdot F_{a_{3}}(x) \cdots F_{a_{k}}(x) \\
& =\sum_{i=0}^{n}(-1)^{i}\left(\begin{array}{c}
i+k-1 \\
k-1
\end{array}\right)\left(\begin{array}{c}
n-i+k-1 \\
k-1
\end{array}\right) L_{n-2 i}(x) .
\end{aligned}
$$

This completes the proofs of all our theorems.

\section{Conclusion}

The main results of this paper are four theorems and five corollaries. Theorem 1 established an identity for the convolution sums of Chebyshev polynomials of the first kind. This improved an early result in [17] and [21]. Theorem 2 simplified the identity in [18] and made it look more concise and beautiful. It must be noted that Theorem 1 and Theorem 2 appear in different forms in other references, such as [3] and [9]. From Theorem 3 and Theorem 4, we can get two corresponding results for Fibonacci polynomials and Lucas polynomials. In addition, in Theorem 4 we have improved a new result in [20]. The five corollaries are just some special cases of our four theorems. These results are actually new contributions to the study of the properties of Chebyshev polynomials and Fibonacci polynomials. Of course, the methods adopted in this paper have some good reference for further study of the properties of general second-order linear recursive sequences. 
Availability of data and materials

Data sharing not applicable to this article as no datasets were generated or analysed during the current study.

\section{Competing interests}

The authors declare that there is no conflict of interests regarding the publication of this paper.

\section{Authors' contributions}

All authors have equally contributed to this work. All authors read and approved the final manuscript.

\section{Publisher's Note}

Springer Nature remains neutral with regard to jurisdictional claims in published maps and institutional affiliations.

Received: 27 December 2019 Accepted: 17 February 2020 Published online: 24 February 2020

\section{References}

1. Borwein, P., Erdèlyi, T.: Polynomials and Polynomial Inequalities. Springer, New York (1995)

2. Yi, Y., Zhang, W.P.: Some identities involving the Fibonacci polynomials. Fibonacci Q. 40, 314-318 (2002)

3. Kim, T., Dolgy, D.V., Kim, D.S.: Representing sums of finite products of Chebyshev polynomials of the second kind and Fibonacci polynomials in terms of Chebyshev polynomials. Adv. Stud. Contemp. Math. 28, 321-336 (2018)

4. Kim, T., Dolgy, D., Kim, D., Seo, J.: Convolved Fibonacci numbers and their applications. Ars Comb. 135, 119-131 (2017)

5. Kim, T., Kim, D.S., Jang, L.-C., Dolgy, D.V.: Representing by several orthogonal polynomials for sums of finite products of Chebyshev polynomials of the first kind and Lucas polynomials. Adv. Differ. Equ. 2019, 162 (2019)

6. Kim, T., Kim, D.S., Dolgy, D.V., Kim, D.: Representation by several orthogonal polynomials for sums of finite products of Chebyshev polynomials of the first, third and fourth kinds. Adv. Differ. Equ. 2019, 110 (2019)

7. Kim, T., Kim, D.S., Dolgy, D.V., Kwon, J.: Sums of finite products of Chebyshev polynomials of the third and fourth kinds. Adv. Differ. Equ. 2018, 283 (2018)

8. Kim, T., Kim, D.S., Dolgy, D.V., Kwon, J.: Representing sums of finite products of Chebyshev polynomials of the first kind and Lucas polynomials by Chebyshev polynomials, Mathematics 7, 26 (2019)

9. Kim, T., Kim, D.S., Jang, L.-C., Dolgy, D.V.: Representation by Chebyshev polynomials for sums of finite products of Chebyshev polynomials. Symmetry 10, 742 (2018)

10. Kim, T., Kim, D.S., Jang, L.-C., Jang, G.-W.: Fourier series for functions related to Chebyshev polynomials of the first kind and Lucas polynomials. Mathematics 6, 276 (2018)

11. Dolgy, D.V., Kim, D.S., Kim, T., Kwon, J.: Connection problem for sums of finite products of Chebyshev polynomials of the third and fourth kinds. Symmetry 10, 617 (2018)

12. Cesarano, C.: Generalized Chebyshev polynomials. Hacet. J. Math. Stat. 43, 731-740 (2014)

13. Cesarano, C.: Integral representations and new generating functions of Chebyshev polynomials. Hacet. J. Math. Stat. 44, 541-552 (2015)

14. Cesarano, C.: Multi-dimensional Chebyshev polynomials: a non-conventional approach. Commun. Appl. Ind. Math. 10, 1-19 (2019)

15. Zhang, W.P.: Some identities involving the Fibonacci numbers. Fibonacci Q. 35, 225-229 (1997)

16. Ma, R., Zhang, W.P.: Several identities involving the Fibonacci numbers and Lucas numbers. Fibonacci Q. 45, 164-170 (2007)

17. Zhang, W.P.: On Chebyshev polynomials and Fibonacci numbers. Fibonacci Q. 40, 424-428 (2002)

18. Zhang, Y.X., Chen, Z.Y.: A new identity involving the Chebyshev polynomials. Mathematics 6, 244 (2018)

19. Zhang, W.P.: Some identities involving the Fibonacci numbers and Lucas numbers. Fibonacci Q. 42, 149-154 (2004)

20. Ma, Y.K., Zhang, W.P.: Some identities involving Fibonacci polynomials and Fibonacci numbers. Mathematics 6, 334 (2018)

21. Wang, S.Y.: Some new identities of Chebyshev polynomials and their applications. Adv. Differ. Equ. 2015,355 (2015)

22. Chen, L., Zhang, W.P.: Chebyshev polynomials and their some interesting applications. Adv. Differ. Equ. 2017, 303 (2017)

23. Li, X.X.: Some identities involving Chebyshev polynomials. Math. Probl. Eng. 2015, Article ID 950695 (2015)

24. Wang, T.T., Zhang, H.: Some identities involving the derivative of the first kind Chebyshev polynomials. Math. Probl. Eng. 2015, Article ID 146313 (2015)

25. Zhang, W.P., Wang, T.T.: Two identities involving the integral of the first kind Chebyshev polynomials. Bull. Math. Soc. Sci. Math. Roum. 108, 91-98 (2017)

26. Chen, Z.Y., Qi, L.: Some convolution formulae related to the second-order linear recurrence sequence. Symmetry 11 , 788 (2019)

27. Kaygisiz, K., Sahin, A.: Determinantal and permanental representations of Fibonacci type numbers and polynomials. Rocky Mt. J. Math. 46, 227-242 (2016)

28. Meng, Y.Y.: A new identity involving balancing polynomials and balancing numbers. Symmetry 11, 1141 (2019)

29. Zhou, S.J., Chen, L.: Tribonacci numbers and some related interesting identities. Symmetry 11, 1195 (2019) 